\title{
Mudanças Climáticas e Suas Relações com o Uso da Terra no Município de Alta Floresta - Amazônia Meridional Brasileira
}

\author{
Katiane da Silva Santos ${ }^{1}$, Beatriz Fátima Alves Oliveira ${ }^{2} \&$ Eliane Ignotti $^{1}$
}

Recebido em 17/07/2020 - Aceito em 07/05/2021

1 Universidade do Estado de Mato Grosso/UNEMAT, Programa de Pós-Graduação em Ciências Ambientais/PPGCA. Brasil. <kati.s.santos@gmail.com, eignotti@uol.com.br>.

2 Escritório Regional da Fundação Oswaldo Cruz - Piauí e Escola Nacional de Saúde Pública Sérgio Arouca/ENSP. Brasil. <beatrizenf@gmail.com>.

\begin{abstract}
RESUMO - A brusca ocupação do norte de Mato Grosso levou à transformação de grandes áreas de transição Cerrado-Amazônia numa das mais importantes regiões de produção agrícola e pecuária do Brasil. Neste estudo buscou-se analisar as mudanças climáticas e sua relação com a mudança do uso da terra em escala local no município Amazônico de Alta Floresta, norte do estado de Mato Grosso, entre 1986 a 2015. Como um estudo exploratório, analisamos as tendências do clima regional por meio de variáveis meteorológicas e ambientais. Foram testadas a relação das temperaturas (máxima, média e mínima) anuais, umidade relativa do ar e precipitação com os indicadores ambientais: área de agropecuária, número de efetivo bovino, área desmatada $e$ número de focos de queimadas. Significativas mudanças climáticas foram observadas em 30 anos para temperatura máxima com aumento de $2,7^{\circ} \mathrm{C}$; para 1986 a 2002 , aumento de $2,9^{\circ} \mathrm{C}$ nas temperaturas máxima e mínima, e diminuição da umidade relativa do ar em 10,7\%; entre 2003 e 2015 , houve diminuição de $2,1 \mathrm{~mm} / \mathrm{m}^{3}$ na precipitação em associação inversa ao desmatamento. $\mathrm{O}$ processo de ocupação da terra por meio do desmatamento e queimadas, seguido da ocupação do solo pela agropecuária, foram determinantes das alterações no clima amazônico de Alta Floresta/MT, sendo evidente o aumento da temperatura e a redução da umidade relativa do ar e da precipitação.
\end{abstract}

Palavras-chave: Conservação dos recursos naturais; incêndios florestais; alta temperatura; agricultura; pecuária.

\section{Climate Change and Its Relations with Land Use in the Municipality of Alta Floresta - Southern Brazilian Amazon}

\begin{abstract}
The sudden occupation of northern Mato Grosso led to the transformation of large areas of transition of the Brazilian Savana-Amazon into one of the most important regions of agricultural and livestock production in Brazil. This study sought to analyze the climate changes and its relationship with land use in a local scale in the Amazon municipality of Alta Floresta, in the north of the state of Mato Grosso, between 1986 and 2015. As an exploratory study, we analyzed regional climate trends by through meteorological and environmental variables. The relation of annual temperatures (maximum, average and minimum), relative humidity and precipitation with environmental indicators were tested: agricultural area, number of cattle, deforested area and number of hot spots. Significant climate changes were observed in 30 years for maximum temperature with an increase of $2.7^{\circ} \mathrm{C}$; for 1986 to 2002 , an increase of $2.9^{\circ} \mathrm{C}$ in the maximum and minimum temperatures, and a decrease in the relative humidity of the air by $10.7 \%$; between $2003-2015$ there was a decrease of $2.1 \mathrm{~mm} / \mathrm{m}^{3}$ in precipitation in an inverse association with deforestation. The process of land occupation through deforestation and biomass burning, followed by land occupation by agriculture and livestock were determinants of changes in the Amazon climate of Alta Floresta/MT, with an evident increase in temperature and a reduction in relative humidity and precipitation.
\end{abstract}

Keywords: Conservation of natural resources; wildfires; high temperature; agriculture; livestock. 


\title{
Cambio Climático y Sus Relaciones con el Uso de la Tierra en el Municipio de Alta Floresta - Amazonia sur Brasileña
}

\begin{abstract}
RESUMEN - La repentina ocupación del norte de Mato Grosso llevó a la transformación de grandes áreas de transición del Cerrado al Amazonas en una de las regiones de producción agrícola y ganadera más importantes de Brasil. Este estudio buscó analizar el cambio climático y su relación con el cambio de uso del suelo a escala local en el municipio amazónico de Alta Floresta, al norte del estado de Mato Grosso, de 1986 a 2015. Como estudio exploratorio analizamos las tendencias climáticas a través de meteorología regional. y variables ambientales. Se probó la relación de temperaturas anuales (máxima, media y mínima), humedad relativa y precipitación con indicadores ambientales: área agrícola, número de ganado, área deforestada y número de incendios. Se observaron cambios climáticos significativos en 30 años para la temperatura máxima con un aumento de $2.7^{\circ} \mathrm{C}$; de 1986 a 2002, un aumento de 2,9 C en las temperaturas máxima y mínima, y una disminución de la humedad relativa del aire en un 10,7\%; entre 2003-2015 hubo una disminución de $2,1 \mathrm{~mm} / \mathrm{m}^{3}$ en la precipitación en una asociación inversa con la deforestación. El proceso de ocupación de la tierra mediante deforestación y quema, seguido de la ocupación del suelo por la agricultura y la ganadería fueron determinantes de los cambios en el clima amazónico de Alta Floresta/MT, con un evidente aumento de temperatura y una reducción de la humedad relativa de la tierra, aire y precipitación.
\end{abstract}

Palabras clave: Conservación de recursos naturales; incendios forestales; alta temperatura; agricultura; ganado.

\section{Introdução}

A relação entre desmatamento e mudanças climáticas é cada vez mais tratada como política interconectada, dado que as florestas exercem importante papel no sequestro de carbono exposto na atmosfera (Buizer et al., 2014) e na regulação do clima em escala global, regional $e$ local (Prevedello et al., 2019). Em escala global, o desmatamento entre 2000 a 2010 foi responsável pelo aquecimento de $0,38^{\circ} \mathrm{C} \pm 0,02^{\circ} \mathrm{C}$ em regiões tropicais e $0,16 \pm 0,01^{\circ} \mathrm{C}$ em regiões de clima temperado (Prevedello et al., 2019).

No Brasil - sétimo emissor de gases de efeito estufa do mundo (GEEs) - 69\% das emissões em 2018 foram decorrentes das mudanças no uso da terra com atividades vinculadas à agricultura $e$ ao desmatamento (SEEG, 2020). Em 2020, apesar da tendência de redução em $6 \%$ das emissões globais devido à paralisação causada pelo novo coronavírus (Covid-19), o Brasil deverá destoar nesse cenário, com a manutenção do aumento da taxa desmatamento nos biomas da Amazônia e do Cerrado e a da perda de $25 \%$ do Pantanal devido aos incêndios florestais em 2020 (INPE, 2020b). Além do crescente desmatamento, o ano também foi marcado por extremos de temperatura em diversos locais, com alerta de risco de morte por hipertermia em grande parte da região CentroOeste e no Norte do Brasil (INMET, 2020).

Grande parte do desmatamento do Brasil deve-se às políticas de desenvolvimento aplicadas no Cerrado e na Amazônia Brasileira desde a década de 60 , e suas causas incluem a interação entre a expansão agrícola, comércio ilegal de madeira, crescimento populacional, projetos de infraestrutura e governança pública que interagem entre si de maneira diferente dependendo da dinâmica temporal e espacial de cada região. Nesse contexto, destaca-se o estado de Mato Grosso, que apresentou, no ano de 2020, o maior número de focos de calor em 2020 e um o maior exportador de grão de soja do país (Faria, 2014).

O estado de Mato Grosso, localizado na Amazônia meridional, com 903 mil km² e cerca de 3 milhões e meio de habitantes, possui em seu território os biomas Amazônia, Cerrado e Pantanal. Atualmente, $40,8 \%$ do Produto Interno Bruto é proveniente da agropecuária (Faria, 2014; IBGE, 2017). A pecuária é importante determinante do desmatamento, cujas queimadas utilizadas como parte do processo de "limpeza das áreas" emite entre vários poluentes o monóxido e o dióxido de carbono ( $\mathrm{CO}$ e $\mathrm{CO}_{2}$, respectivamente). Soma-se a isso a criação bovina, responsável pela maior emissão mundial de metano $\left(\mathrm{CH}_{4}\right)$ e óxido nitroso $\left(\mathrm{N}_{2} \mathrm{O}\right)$ (Bailey et al., 2014).

A marcha para Oeste, iniciada em 1938, teve importante impacto no desenvolvimento regional do norte do Mato Grosso, e propunha colonizar o Centro-Oeste e Amazônia. Como projeto governamental, tinha por objetivo "integrar $e$ consolidar a nação". Essa política tornou-se o eixo central de integração. Somente a partir da década 
de 1970, houve intenso processo migratório, em especial para a região norte de Mato Grosso, cujos incentivos do governo federal que propiciaram recursos para o cultivo de lavouras (Raminger et al., 2008; Calonga, 2015).

O município de Alta Floresta foi criado a partir do projeto INDECO S/A (Integração, Desenvolvimento e Colonização) concomitante à campanha "Integrar para não entregar" do Governo Federal. A pavimentação da BR 163, que ligava o município ao Sudeste e Sul do país, facilitou o seu crescimento. A economia variou ao longo do tempo, tendo a extração vegetal, na década de 1970 e 1980, partindo para a agricultura. Atualmente, destaca-se como um dos maiores produtores de gado de corte do estado (SEPLAG, 2014; IBGE, 2019). De acordo com o IBGE (2017), Alta Floresta foi emancipada como município em 1979 , com $52.572 \mathrm{~km}^{2}$. A partir de 1986, teve início o desmembramento de distritos e a emancipação dos municípios de Paranaíta, Apiacás; em 1991, de Nova Monte Verde e Nova Bandeirantes; e, em 1994, do município de Carlinda. Alta Floresta, localizado na região geográfica imediata de Sinop, sendo os municípios pertencentes a essa região, grandes produtores agropecuários, representando vultosa
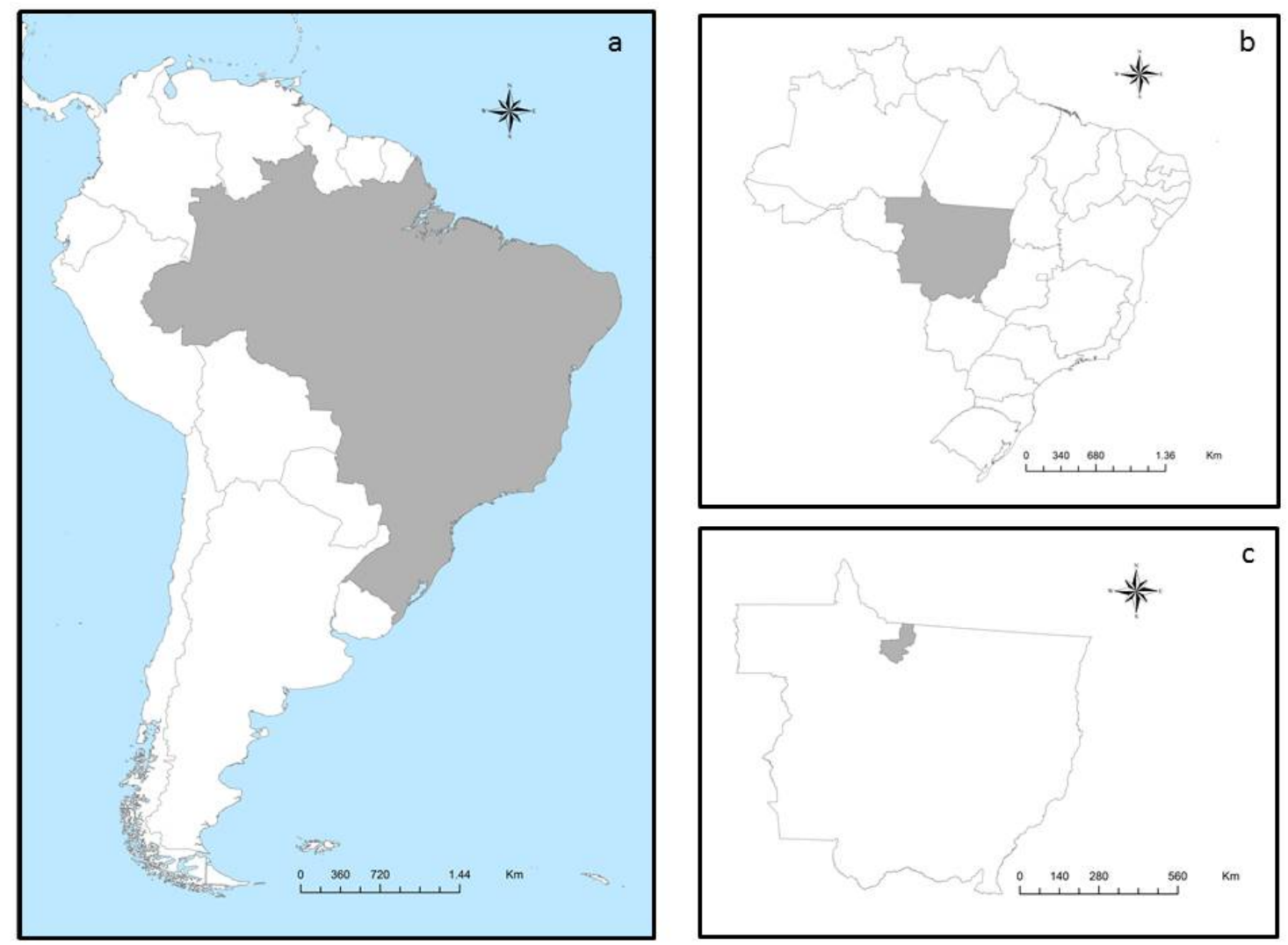

Figura 1 - Localização geográfica do município de Alta Floresta, estado de Mato Grosso - Brasil. 
A temperatura máxima, média e mínima, umidade e precipitação foram obtidas do conjunto de dados de reanálises ERA-Interim gerados pelo Centro Europeu de Previsão do Tempo (European Centre for Medium-Range Weather Forecasts ECMWF), com resolução $0,125^{\circ} \times 0,125^{\circ}$ para o período de 1986 a 2015. Esses dados foram modelados a partir de dados observacionais disponibilizados no formato NetCDF (Network Common Data Form). Os arquivos nesse formato são matrizes que armazenam metadados em um mesmo arquivo constituído pelas variáveis tempo, latitude e longitude (em pontos de grade) $e$ as variáveis meteorológicas. Assim, um arquivo NetCDF que contém informações diárias de temperatura durante um mês contém 30 grids. Cada célula ou pixel da imagem remete um valor da variável, e equivale a um quadrado com lado correspondente a resolução (ECMWF, [s.d.]) (Dee et al., 2011). Foi realizado um cálculo de média anual para os dados meteorológicos, pois o banco de dados inicial era constituído por observações diárias.

Os dados de área de agropecuária foram extraídos do MapBiomas, uma plataforma de rede de colaboração entre especialista em biomas, usos da terra, sensoriamento remoto, Sistema de Informação Geográfica (SIG) e ciência da computação. O MapBiomas utiliza processamento em nuvem e classificadores automatizados desenvolvidos e operados a partir da plataforma Google Earth Engine para gerar uma série histórica de mapas anuais de cobertura e uso da terra do Brasil. Os dados referentes ao efetivo bovino são oriundos do IBGE que provém do censo agropecuário, resultado no número unitário de cabeças do bovino por ano em estudo.

Foram coletados dados área desmatada na plataforma Projeto de Estimativa do Desflorestamento da Amazônia (PRODES) do Instituto Nacional de Pesquisas Espaciais (INPE). O PRODES realiza o monitoramento por satélite do desmatamento por corte raso na região da Amazônica e produz, desde 1988, as taxas anuais de desmatamento na região. As taxas anuais são estimadas a partir dos incrementos de desmatamento identificados em cada imagem de satélite que cobre a Amazônia brasileira.

Dados sobre focos de queimadas foram coletados do INPE que realiza a contagem de focos por meio de satélites. A relação foco $\mathrm{x}$ queimada não é direta nas imagens de satélite. Um foco indica a existência de fogo em um elemento de resolução da imagem (pixel), que varia de $375 \mathrm{~m} \times 375 \mathrm{~m}$ até $5 \mathrm{~km} \times 4 \mathrm{~km}$, dependendo do satélite. Nesse pixel pode haver uma ou várias queimadas distintas que a indicação será de um único foco. Se uma queimada for muito extensa, ela será detectada em alguns pixeis vizinhos, ou seja, vários focos estarão associados a uma única grande queimada. A Tabela 1 apresenta as variáveis ambientais e climáticas, bem como as respectivas fontes de dados.

Tabela 1 - Fonte de dados das variáveis ambientais e climáticas.

\begin{tabular}{|l|l|}
\hline Indicador & Fonte \\
\hline Temperatura (máxima, média e mínima) & ERA-IECMWF \\
\hline Umidade relativa do ar & ERA-IECMWF \\
\hline Precipitação & ERA-IECMWF \\
\hline Desmatamento & PRODES/INPE \\
\hline Focos de queimadas & INPE \\
\hline Área de agropecuária & MapBiomas \\
\hline Número de efetivo bovino & IBGE \\
\hline
\end{tabular}

Ao analisar as tendências dos dados climáticos durante o período de 1986 a 2015, definiu-se como ponto de corte o ano de 2002, em razão da inflexão observada para a maioria das variáveis.
Por esta razão as análises foram realizadas para o período completo com 30 anos (de 1986 a 2015) e para dois períodos, expressos em 17 anos (1986 e 2002) e 13 anos (2003 e 2015). 
As variáveis relacionadas ao uso da terra foram padronizadas em índices em razão das distintas unidades de medida, bem como para possibilitar as análises combinadas de desmatamento e focos de calor e de áreas de agropecuária e de efetivo bovino. Para a construção dos índices, utilizou-se a equação descrita a seguir.

$$
\text { Índices }=1-\frac{(\text { valor máximo }- \text { valor observaodo })}{\text { (valor máximo }- \text { valor mínimo) }}
$$

Assim, área agropecuária, efetivo bovino, desmatamento $e$ focos de queimadas tiveram seus índices construídos e a seguir, denominados de INDAGRO, INDBOV, INDDESM, INDFOCO, respectivamente.

Pela relação, os índices de área agropecuária foram unidos ao de efetivo bovino, por meio de média, denominado por AGROBOV, bem como entre desmatamento e focos de queimadas denominado por DESMFC.

Sendo,

- INDAGRO: o índice da área agropecuária;

- INDBOV: o índice do número do efetivo bovino;

- INDDESM: o índice de área de desmatamento;

- INDFOCO: o índice de números de focos de queimadas;

- AGROBOV: índice entre área de agropecuária e número de efetivo bovino;

- DESMFC: índice entre área de desmatamento e número de focos de queimadas.

A partir da análise de regressão linear simples, testou-se a interação entre os indicadores resposta, temperatura máxima, temperatura mínima, temperatura média, umidade $e$ precipitação com os indicadores relativos à agropecuária, efetivo bovino, desmatamento e focos de queimadas, bem como com os seus respectivos índices. Foi também analisada a tendência de cada indicador em cada período ao nível de significância de 5\%.

Os programas computacionais utilizados foram o $R$ versão 3.6.1 (R. Core Team, 2020) para análise de regressão e para a análise de tendência, empregou-se um modelo de regressão por pontos de inflexão (joinpoint regression analysis). Esse modelo verifica se uma linha com múltiplos segmentos é estatisticamente melhor para descrever a evolução temporal de um conjunto de dados em detrimento de uma linha reta ou com menos segmentos. Também permite identificar a tendência do indicador (se estacionária, crescente ou decrescente).

\section{Resultados}

Ao comparar o ano inicial de estudo (1985) ao último ano (2015), para os indicadores, é possível observar que a área desmatada inicial foi de $1.817 \mathrm{~km}^{2}$ e $4.946,1 \mathrm{~km}^{2}$, respectivamente; os números de focos de queimadas sofreram aumento de 62 para 1.489; a área de agropecuária inicial era de $801,3 \mathrm{~km}^{2}$ chegando a $4.253,5 \mathrm{~km}^{2}$; o número de efetivo bovino aumentou de 80.507 e aumentou para 716.438; a média da temperatura máxima em 1986 era de $27,39^{\circ} \mathrm{C}$ e $30,06^{\circ} \mathrm{C}$ em 2015 ; a média da temperatura média aumentou de $24,19^{\circ} \mathrm{C}$ para $26,22^{\circ} \mathrm{C}$; a média da temperatura mínima de 21,07 para 22,02; a média da umidade relativa do ar diminuiu de $85,22 \%$ para $75,23 \%$ e a precipitação diminuiu de $5,9 \mathrm{~mm} / \mathrm{m}^{3}$ para $4,69 \mathrm{~mm} / \mathrm{m}^{3}$.

No período de 30 anos (de 1986 a 2015) em estudo, as médias anuais das temperaturas máxima, média e mínima apresentaram tendência linear ascendente e a umidade relativa do ar e precipitação, descendente. Destaca-se a temperatura máxima, verificando aumento significante $2,7^{\circ} \mathrm{C}$ na média anual no período em estudo. Nos primeiros 17 anos da série (de 1986 a 2002) o incremento verificado é maior quando comparado ao segundo período de 13 anos (de 2003 a 2015). Alta Floresta apresentou tendência de aumento de $0,17^{\circ} \mathrm{C}$ na média anual de temperatura máxima entre os anos de 1986 a 2002, aumento de $0,10^{\circ} \mathrm{C}$ na temperatura média, $0,17^{\circ} \mathrm{C}$ na temperatura mínima. Concomitantemente houve redução de $0,63 \%$ na umidade relativa do ar e $0,11 \mathrm{~m}^{3} / \mathrm{mm}$ na precipitação. Entre 2003 e 2015, verificouse aumento foi de $0,11^{\circ} \mathrm{C}$ para temperatura máxima, $0,09^{\circ} \mathrm{C}$ na temperatura média $e$, aumento não significativo da temperatura mínima, concomitante a diminuição de $0,49 \%$ na umidade relativa do ar e $0,16 \mathrm{~m}^{3} / \mathrm{mm}$ na precipitação (Tabela 2). 
Tabela 2 - Medidas descritiva e tendência das temperaturas, umidade relativa do ar e da precipitação no período de 1986-2015. Alta Floresta, Mato Grosso.

\begin{tabular}{|c|c|c|c|c|c|}
\hline & Média & Máximo & Mínimo & \multicolumn{2}{|c|}{ Tendência } \\
\hline \multicolumn{6}{|l|}{ Alta Floresta } \\
\hline \multicolumn{6}{|c|}{ Temperatura máxima } \\
\hline $1986-2015$ & 28,88 & 31,21 & 27,39 & $0,09 * * *$ & nom \\
\hline 1986-2002 & 28,45 & 31,21 & 27,39 & $0,17^{* * *}$ & $\sim$ \\
\hline $2003-2015$ & 29,46 & 30,23 & 28,21 & $0,11^{* * *}$ & $\sim$ \\
\hline \multicolumn{6}{|c|}{ Temperatura média } \\
\hline $1986-2015$ & 25,23 & 26,34 & 24,19 & $0,06^{* * * *}$ & mens \\
\hline $1986-2002$ & 24,91 & 26,34 & 24,19 & $0,10 * * *$ & and \\
\hline 2003-2015 & 25,63 & 26,32 & 25,09 & $0,09 * * *$ & m \\
\hline \multicolumn{6}{|c|}{ Temperatura mínima } \\
\hline 1986-2015 & 21,65 & 22,20 & 21,07 & $0,02 * *$ & moner \\
\hline $1986-2002$ & 21,54 & 22,20 & 21,07 & $0,17^{* * *}$ & $\sim m$ \\
\hline 2003-2015 & 21,80 & 22,20 & 21,49 & 0,11 & $\sim \sim$ \\
\hline \multicolumn{6}{|l|}{ Umidade } \\
\hline 1986-2015 & 79,39 & 88,07 & 70,93 & $-0,03$ & nomm \\
\hline 1986-2002 & 81,40 & 88,07 & 70,93 & $-0,63 * * * *$ & nn \\
\hline $2003-2015$ & 76,76 & 80,41 & 73,25 & $-0,49$ ***** & $\sim$ \\
\hline \multicolumn{6}{|l|}{ Precipitação } \\
\hline $1986-2015$ & 5,17 & 6,76 & 2,93 & $-0,03$ & nom \\
\hline 1986-2002 & 5,11 & 5,93 & 2,93 & $-0,11^{* *}$ & $q$ \\
\hline 2003-2015 & 5,26 & 6,76 & 4,13 & $-0,16^{* *}$ & $\sim$ \\
\hline
\end{tabular}

$\mathrm{p}<0,001^{* * *}, \mathrm{p}<0,01 * *, \mathrm{p}<0,05 *$

Os resultados sintetizados mostram de forma mais expressiva as mudanças no clima ocorridos nos primeiros 17 anos da série (1986-2002). Para temperatura máxima o aumento foi de $2,9^{\circ} \mathrm{C}$, para temperatura média de $1,7^{\circ} \mathrm{C}$ e para temperatura mínima de $2,9^{\circ} \mathrm{C}$, a diminuição da umidade relativa do ar foi de $10,7 \%$ e para precipitação de $1,9 \mathrm{~mm} / \mathrm{m}^{3}$ (Tabela 3 ).

Tabela 3 - Síntese das tendências das variáveis meteorológicas no período de 1986 a 2015, no município de Alta Floresta, no Mato Grosso.

\begin{tabular}{|l|c|c|c|c|c|}
\hline & TMAX & TMED & TMIN & UMID & PREC \\
\hline Alta Floresta & & & & & n.s. \\
\hline $1986-2015$ & $2,7^{\circ} \mathrm{C}$ & $1,8^{\circ} \mathrm{C}$ & $0,6^{\circ} \mathrm{C}$ & n.s. & $-1,9 \mathrm{~mm} / \mathrm{m}^{3}$ \\
\hline $1986-2002$ & $2,9^{\circ} \mathrm{C}$ & $1,7^{\circ} \mathrm{C}$ & $2,9^{\circ} \mathrm{C}$ & $-10,70 \%$ & $-2,1 \mathrm{~mm} / \mathrm{m}^{3}$ \\
\hline $2003-2015$ & $1,4^{\circ} \mathrm{C}$ & $1,2^{\circ} \mathrm{C}$ & n.s & $-6,40 \%$ & \\
\hline
\end{tabular}

*n.s.: não significante 
Foi realizada análise de regressão linear da variação da temperatura, umidade e indicadores ambientais. Em Alta Floresta no período analisado (de 1986 a 2015), a temperatura mínima e índice de focos de queimadas, foi a única associação que apresentou significância. No primeiro recorte que é equivalente aos primeiros anos (de 1986 a 2002) o desmatamento associa-se ao aumento da temperatura máxima; o aumento no índice de desmatamento $e$ focos de queimadas associam-se o aumento da temperatura média, bem como a diminuição nesse mesmo índice responde a queda da umidade. Para os 13 anos seguintes (de 2003 a 2015) apenas os focos de queimadas estão associados ao aumento da temperatura mínima (Tabela 4).

Tabela 4 - Análise de regressão linear da variação de temperatura, umidade e indicadores no período de 1986 a 2015, no município de Alta Floresta, Mato Grosso.

\begin{tabular}{|c|c|c|c|}
\hline Variáveis climáticas & Indicadores e Índices ambientais & $\beta$ & p valor \\
\hline \multicolumn{4}{|l|}{ Alta Floresta } \\
\hline \multicolumn{4}{|l|}{$1986-2015$} \\
\hline Temp Min & $\mathrm{N}^{\circ}$ de Focos de Queimadas & 0,000 & 0,044 \\
\hline \multicolumn{4}{|l|}{ 1986-2002 } \\
\hline Temp Max & Desmatamento $\mathrm{km}^{2}$ & 0,020 & $<0.001$ \\
\hline Temp Méd & Desmatamento km² & 0,006 & 0,004 \\
\hline Temp Méd & $N^{\circ}$ de Focos de Queimadas & 0,001 & 0,005 \\
\hline Temp Méd & Índice Desmatamento e Focos de Queimadas & 2,700 & 0,003 \\
\hline Umidade & Desmatamento km² & $-0,060$ & $<0.001$ \\
\hline Umidade & $N^{\circ}$ de Focos de Queimadas & $-0,006$ & 0,008 \\
\hline Umidade & Índice Desmatamento e Focos de Queimadas & $-2,690$ & 0,003 \\
\hline \multicolumn{4}{|l|}{ 2003-2015 } \\
\hline Temp Mín & $N^{\circ}$ de Focos de Queimadas & 0,000 & 0,049 \\
\hline
\end{tabular}

\section{Discussão}

Este é o primeiro estudo a analisar a relação do clima $e$ indicadores ambientais ocorridos no município de Alta Floresta, Amazônia meridional. Os achados mostram aumentos significativos nas temperaturas máxima, média e mínima concomitante a diminuição da umidade relativa do ar e da precipitação nos 30 anos em estudo (19862015). As mudanças no clima em Alta Floresta mostraram-se associadas ao processo de ocupação territorial com aumento das emissões estimadas de $\mathrm{CO}_{2}$ e, principalmente, com a ampliação da área desmatada e registros de focos de queimadas, que antecedem e ocorrem concomitantemente com o avanço da agropecuária.
Foi possível identificar que o ano de 2002 teve um importante ponto de inflexão e ao analisar os dois períodos separadamente (1986-2002 e 2003-2015). No primeiro período analisado (1986-2002) houve aumentos da temperatura e diminuição da umidade relativa do ar mais significativos quando comparado ao segundo período (2003-2015). Essas alterações no primeiro período tiveram o desmatamento como principal indicador ambiental relacionado. Até 2002 estima-se que uma área de 4.245,7 km² havia sido desmatada. Essa relação não foi observada nos 13 anos subsequentes (2003-2015) que apresentou um incremento de $700,4 \mathrm{~km}^{2} \mathrm{de}$ área desmatada. 
O desmatamento da Amazônia vem aumentando desde 1991 em razão de diversos fatores. No entanto os fins agropecuários, principalmente no Mato Grosso são os mais evidentes (Fearnside, 2020). Sivestrini et al. (2011) já alertava que as mudanças climáticas combinadas com o desmatamento contínuo e a pavimentação de estradas poderiam degradar a Floresta Amazônica, não apenas aumentando a ocorrência de incêndios, mas contribuindo para a propagação do fogo nas florestas úmidas do noroeste da Amazônia, que são atualmente resistente ao fogo.

O desmatamento entre 2000 a 2010 já promoveu aquecimento global de $0,38^{\circ} \mathrm{C}$, mas essa relação é proporcional a área desmatada, como por exemplo, em áreas que perderam $50 \%$ da cobertura florestal, o aumento médio de $1,08^{\circ} \mathrm{C}$ e em áreas com $100 \%$ de desmatamento, o aumento pode chegar a $2^{\circ} \mathrm{C}$. No Brasil, mais especificamente na Amazônia Brasileira, em um cenário de taxas de desmatamento semelhantes às atuais, a temperatura poderá subir em média $1,45^{\circ} \mathrm{C}$ entre 2010 a 2050. Esse aumento foi vinculado com o aumento do albedo - coeficiente de reflexão de uma superfície - $e$ a redução da evapotranspiração (Prevedello et al., 2019). A combinação entre redução de evapotranspiração e aumento da temperatura acarreta um declínio geral da umidade relativa do ar, com redução de $10 \%$ a $23 \%$ em média sobre em cenários com desmatamento de moderado à moderado-alto em áreas de proteção, respectivamente (Sales et al., 2020). Além disso, essas mudanças no clima local podem, assim, influenciar na circulação regional e global, nos padrões de precipitação (Jacson et al., 2008) e no transporte de umidade para dentro e fora da região, afetando os níveis dos rios Amazônicos (Marengo \& Souza Jr, 2018).

$\mathrm{Na}$ Amazônia Brasileira, o crescente desmatamento, aliado às mudanças climáticas tem influenciado no ciclo hidrológico da região $e$ ameaçado a sustentabilidade da floresta em níveis considerados críticos ("tipping point") (Lovejoy \& Nobre, 2018). Ao atingir esse ponto de inflexão e com as mudanças nos padrões de precipitação, a floresta poderá deixar de produzir chuva suficiente para se sustentar degradando-se lentamente em uma savana mais seca e contribuindo para a liberação de toneladas de carbono na atmosfera (Lovejoy \& Nobre, 2018).
As mudanças no padrão de chuvas na região, com redução dos volumes de precipitação, podem ter implicações sobre a agricultura. Com o estresse hídrico na região, sobretudo durante as secas, a produtividade agrícola e de pastagens podem ser impactadas drasticamente, com redução de 28 a $33 \%$ em áreas de pastagens e de $25 \%$ na produção de soja, podendo chegar em a $60 \%$ em alguns locais (PBMC, 2014; BRASIL, 2015). Portanto, a priorização de um modelo econômico $e$ de desenvolvimento que incentive a remoção das florestas pode levar à custos econômicos ainda maiores.

Além do desmatamento, as mudanças no clima entre 1986 a 2002, sobretudo o aumento da temperatura e a redução da umidade, foram relacionadas ao número de focos de calor. Entre o período de 2000 a 2015, o número de focos de queimada foi relacionado positivamente com a temperatura mínima temperatura mínima. De acordo com Nobre (2007), a floresta Amazônica possuía certa impermeabilidade ao fogo. No entanto, a diminuição da umidade pelo desmatamento, a fragmentação florestal $e$ a prática agrícola podem ter influenciado nesta proteção natural de forma abrupta, aumentando as condições favoráveis aos incêndios florestais, com considerados rearranjos nos ecossistemas $e$ possíveis redistribuição de biomas. A teleconexão permite a propagação de impactos climáticos em áreas geograficamente remotas, e mesmo perturbações relativamente pequenas da cobertura do solo nos trópicos podem impactar em latitudes altas (Lawrence \& Vandecar, 2014).

Além da perda de biodiversidade, os incêndios florestais levam a emissão de poluentes atmosféricos que resultam em problemas de saúde para a população. Ignotti et al. (2010) encontrou associação entre a ocorrência de doenças respiratórias na Amazônia brasileira e a poluição atmosférica, principalmente para crianças e idosos, por pertencerem a faixa etária mais vulnerável e durante o período de estiagem. As tendências estimadas neste estudo mostram que o aumento da temperatura e a redução da umidade relativa do ar e das precipitações são condições climáticas favoráveis para exacerbar eventos como os incêndios florestais.

Outra problemática que pode surgir é a competição por terras, levando ao aumento dos preços dos alimentos e intensificação adicional 
(por exemplo, uso de fertilizantes e água) com implicações na poluição da água e do ar $e$ na perda adicional de biodiversidade. Tais consequências colocariam em risco a capacidade das sociedades de alcançar muito dos Objetivos de Desenvolvimento Sustentável (ODS) que dependem da terra (IPCC, 2019).

Fearnside (2006) sugeriu que a principal solução para a diminuição de emissão de GEE e do desmatamento, deverá ocorrer por meio de políticas públicas que freiem o ato. Após pico de desmatamento em 2004 e 2005, impulsionado pela expansão da produção de soja e carne, o desmatamento na Amazônia começou a cair após uma série de ações governamentais como o fortalecimento dos órgãos ambientais de fiscalização e criação de novas áreas protegidas na Amazônia, incluindo reservas indígenas e unidades de uso sustentável. No entanto, a partir de 2013, os números crescentes do desmatamento no Cerrado, na Amazônia e, mais recentemente, no Pantanal tem preocupado a comunidade científica e vários setores da sociedade (INPE, 2020b). Além disso, para Rojas-Downing et al. (2017) a utilização de culturas mistas e diversidade de animais pode ser uma medida de mitigação promissora, pois ao diversificar, a tolerância à variabilidade climática, doenças e surtos de pragas será melhorada.

Em relação ao armazenamento de carbono, a atuação das florestas depende das características de cada bioma. Na Caatinga, a máxima efetividade de armazenamento de carbono é sazonal e ocorre nos meses mais úmidos (Mendes et al., 2020) e no Pantanal o estoque de carbono no solo está condicionado pela sazonalidade e é dependente da fitofisionomia local (Mendes et al., 2015). Enquanto a Floresta Amazônica desempenha um importante papel no ciclo de carbono planetário (Nobre et al., 2007) e na regulação climática, especialmente na temperatura e no ciclo hidrológico em escala global, regional e local (Prevedello et al., 2019).

\section{Conclusão}

O estudo limitou-se a avaliar o comportamento das mudanças climáticas em Alta Floresta, por meio das variáveis climáticas mais usuais. Ressalta-se que mudanças regionais e globais também influenciam climas locais. No entanto, trata-se de exemplo de área bruscamente transformada na Amazônia meridional. Cabe o aprofundamento desse estudo da influência das mudanças na região, bem como, com a inclusão de outras variáveis explicativas.

Conclui-se que o processo de ocupação da terra por meio do desmatamento e queimadas, seguido da ocupação do solo pela agropecuária foram determinantes das alterações no clima Amazônico de Alta Floresta/MT, sendo evidente o aumento da temperatura e a redução da umidade relativa do ar e da precipitação.

\section{Agradecimentos}

Este estudo foi financiado, em parte, pela Coordenação de Aperfeiçoamento de Pessoal de Nivel Superior Brasil (CAPES), Código de Financiamento 001, pela bolsa de estudos, bem como pela parceria com a Rede Clima Brasil, $\mathrm{n}^{\circ}$ 550022/2014-7 e FINEP/Rede Clima (01.13.0353.00).

\section{Referências}

Bailey R, Froggatt A \& Wellesley L. Livestock - Climate Change's Forgotten Sector. Global Public Opinion on Meat and Dairy Consumption. Energy, Environment and Resources | December 2014. Chatam house.

BRASIL. Ministério do Meio Ambiente, B. Plano Nacional de Adaptação VolumeII à Mudança do Clima. Plano Nac. Adapt. à Mudança do Clima Vol. II II, 394 (2015).

Buizer M, Humphreys D \& Jong W. Climate Channge and Desflorestation: The evolution of an intersecting policy domain. Environmental Science \& Policy. Volume 35, January 2014, Pages 1-11. Elsevier. < https://doi.org/10.1016/j.envsci.2013.06.001>. Acesso em: 04/03/2020.

Calonga MD. A Marcha para o Oeste e os intelectuais em Mato Grosso: política e identidade. Revista Espaço Acadêmico - No 168 - maio 2015. < http://periodicos. uem.br/ojs/index.php/EspacoAcademico/article/ view/26438 > . Acesso em: 09/06/2018.

Carmo RL, Vazquez FF \& Camargo K. Agronegócio e Dinâmica Populacional: A Soja e os Frigoríficos em Mato Grosso. Boletim regional, urbano e ambiental | 17 | jul.-dez. 2017 ipea. <http://repositorio. ipea.gov.br/bitstream/11058/8154/1/BRU_n17_ Agroneg\%C3\%B3cio.pdf>. Acesso em: 27/05/2020.

Carvalho JLN et al. Potencial de sequestro de carbono em diferentes biomas do Brasil. Rev. Bras. Ciênc. Solo, 
Viçosa, v. 34, n. 2, p. 277-290, Apr. 2010. < https://doi. org/10.1590/S0100-06832010000200001>. Acesso em: 08/10/2020.

De Sales F, Santiago T, Biggs TW, Mullan K, Sills EO \& Monteverde C. Impacts of Protected Area Deforestation on Dry-season Regional Climate in the Brazilian Amazon. Journal of Geophysical Research: Atmospheres (2020). <doi:10.1029/2020jd033048>. Acesso em: 20/10/2020.

Dee DP et al. The ERA-Interim reanalysis: configuration and performance of the data assimilation system, 2011. Q.J.R. Meteorol. Soc., 137: 553-597. <https://rmets. onlinelibrary.wiley.com/doi/full/10.1002/qj.828>. Acesso em: 27/05/2019.

ECMWF (European Centre for Medium-Range Weather Forecasts) [s.d.]. Centro Europeu de Previsão do Tempo. <http://apps.ecmwf.int/datasets/data/interimfull-daily/levtype $=$ sfc/ $>$. Acesso em: 28/05/2019.

Faria AMM. Perspectivas para o desenvolvimento de Mato Grosso. Rio de Janeiro: Banco Nacional de Desenvolvimento Econômico e Social, 2014. p. [394]-423. <http://web.bndes.gov.br/bib/jspui/ handle/1408/7521 > . Acesso em: 08/06/2018.

Fearnside PM. (ed.) 2020. Destruição e Conservação da Floresta Amazônica, Vol. 1. Editora do INPA, Manaus. 368 p. (no prelo). <http://portal.inpa.gov.br/ index.php/2017-09-04-22-13-15/publicacoes-inpa > . Aceso em: 26/05/2020.

IBGE (Instituto Brasileiro de Geografia e Estatística). Divisão Regional do Brasil, 2017. < https://ww2.ibge. gov.br/home/geociencias/geografia/default_div_int. shtm?c=1\# > Acesso em: 15/07/2018.

IBGE (Instituto Brasileiro de Geografia e Estatística). IBGE CIDADES, 2019. https://cidades.ibge.gov.br/. Acesso em: 28/03/2020.

Ignotti E, Valente JG, Longo KL, Freitas SR, Hacon SS \& Netto PA. Impact on human health of 2 matter emitted from burnings in the Brazilian Amazon region. Rev Saude Publica. 2010; 44(1): 121-130. doi:10.1590/ s0034-89102010000100013. Acesso em: 28/05/2020.

INPE (Instituto Nacional de Pesquisas Espaciais). Queimadas. Acesso em: <http://www.inpe.br/ queimadas/portal>. Acesso em: 20//11/2018.

INPE. Instituto Nacional de Pesquisas Espaciais. Queimadas - Monitoramento dos Focos Ativos por Estado. 2020b. <http://queimadas.dgi.inpe.br/ queimadas/portal-static/estatisticas_estados/>. Acesso em: 19/10/2020

IPCC (Intergovernmental Panel of Climate Change) 2014. Synthesis Report. Contribution of Work Groups I, II and III to the Fifth Assesment Report of the Intergovernmental Panel of Climate Change [Core
Writing Team; Pachauri RK \& Meyer LA (eds.)]. IPCC Geneve, Switzerland, 151 pp. <https://issuu.com/ unipcc/docs/syr_ar5_final_full_wcover $>$. Acesso em: 29/03/2020.

IPCC (The Intergovernmental Panel on Climate Change). Special Report: Special Report an Climate Change and Land. Working Group III. 2019. < https:// www.ipcc.ch/srccl/chapter/chapter-1/>. Acesso em: 20/04/2020.

Jackson CS, Sen MK, Huerta G, Deng Y \& Bowman KP. Error reduction and convergence in climate prediction. J Climate. 2008; 21: 6698-6709. 10.1175/2008JCLI2112.1 [CrossRef] [Google Scholar]

Lawrance D \& Vandecar K. Effects of tropical drestation on climate and agricuture. Department of Environmental Sciences, University of Virginia Clark Hall, 291 McCormick Road, Charlottesville, Virginia 22904-4123, USA. NATURE CLIMATE CHANGE |VOL 5| JANUARY 2015. < doi:10.1038/nclimate2430>. Acesso em: 06/03/2020.

Lovejoy TE \& Nobre C. Amazon Tipping Point. Science Advances 2018; 4(2): eaat2340.

Marengo JA \& Souza Jr C. Mudanças climáticas: Impactos e cenários para a Amazônia. São Paulo, 2018. <https://www.conectas.org/wp/wp-content/ uploads/2018/12/Relatorio_Mudancas_ClimaticasAmazonia.pdf > . Acesso em: 19/10/2020.

Mello JM et al. Dinâmica dos atributos físico-químicos $e$ variação sazonal dos estoques de carbono no solo em diferentes fitofisionomias do pantanal norte matogrossense. Rev. Árvore. < https://doi.org/10.1590/010067622015000200012 >. Acesso em: 09/10/2020.

Mélo AS, Justino F, Lemos CF, Sediyama G \& Ribeiro G. Suscetibilidade do Ambiente a Ocorrências de Queimadas sob Condições Climáticas Atuais e de Futuro Aquecimento Global. Revista Brasileira de Meteorologia, v.26, n.3, 401-418, 2011. Universidade Federal de Viçosa (UFV), Viçosa, MG, Brasil.

Mendes KR et al. Seasonal variation in net ecosystem $\mathrm{CO} 2$ exchange of a Brazilian seasonally dry tropical forest. Scientific Reports, 10, 9454 (2020). < https:// doi.org/10.1038/s41598-020-66415-w>. Acesso em 07/10/2020.

Naqvi SMK \& Sejian V. Global Climate Change: Role of Livestock. Division of Physiology and Biochemistry, Central Sheep and Wool Research Institute, Avikanagar (Via-Jaipur), Rajasthan-304 501, India. Asian Journal of Agricultural Sciences 3(1): 19-25, 2011. <https:// www.researchgate.net/publication/49605076>. Acesso em: 09/03/2020.

Nobre CA, Sampaio G \& Salazar L. Mudanças climáticas e Amazônia. Cienc. Cult. [Internet]. 2007 
Sep [cited 2020 Oct 19]; 59(3): 22-27. Available $<$ http://cienciaecultura.bvs.br/scielo.php?script $=$ sci arttext\&pid=S0009-67252007000300012\&lng $=e n>$. Acesso em: 20/10/2020.

Nobre CA, Sampaio G \& Salazar L. Mudanças Climáticas e Amazônia, 2007. Centro de Previsão de Tempo e Estudos Climáticos (CPTEC), Instituto Nacional de Pesquisas Espaciais (Inpe). <http:// cienciaecultura.bvs.br/scielo.php? script $=$ sci_arttext \&pid=S0009-67252007000300012 >. Acesso em: 01/02/2020.

PBMC. Painel Brasileiro de Mudanças Climáticas in Mitigação das mudanças climáticas. Contribuição do Grupo de Trabalho 3 do Painel Brasileiro de Mudanças Climáticas ao Primeiro Relatório da Avaliação Nacional sobre Mudanças Climáticas 463 (2014).

Prevedello JA, Winck GR, Weber MM, Nichols E \& Sinervo B. Impacts of forestation and deforestation on local temperature across the globe. PLoS One. 2019 Mar 20; 14(3): e0213368. <doi: 10.1371/ journal.pone.0213368>. PMID: 30893352; PMCID: PMC6426338.

PRODES (Programa de Cálculo do Desflorestamento da Amazônia). < http://www.obt.inpe.br/OBT/assuntos/ programas/amazonia/prodes. $>$ Aceso em: 22/11/2018.

Projeto MapBiomas - Coleção 4 da Série Anual de Mapas de Cobertura e Uso de Solo do Brasil. < https:// mapbiomas.org > . Acesso em: 11/09/2020.

R Core Team (2020). R: A language and environment for statistical computing. R Foundation for Statistical Computing, Vienna, Austria. <https://www.R-project. org/. > Acesso em: 20/10/2020.

Rojas-Downing MM, Nejadhashemi AP, Harrigan T \& Woznicki SA. Climate change and livestock: Impacts, adaptation, and mitigation. Climate Risk Management 2212-0963/2017 Published by Elsevier B.V. Department of Biosystems and Agricultural Engineering, Michigan State University, 524 S. Shaw Lane, Room 225, East Lansing, MI 48824, USA. http://dx.doi.org/10.1016/j. crm.2017.02.001. Acesso em: 07/03/2020.

Schlesing S. Soja: o grão que cresceu demais e os impactos deste crescimento sobre a sociedade e o meio ambiente. 2004. Rio de Janeiro: FASE, 148 p. https://fase.org.br/wp-content/uploads/2006/11/ $\mathrm{O}+\mathrm{gr} \% \mathrm{C} 3 \% \mathrm{~A} 3 \mathrm{o}+\mathrm{que}+$ cresceu + demais.pdf. Acesso em: 28/03/2020.

SEEG. Sistema de Estimativas de Emissões e Remoções de Gases de Efeito Estufa. Novas estimativas do SEEG Brasil - Sistema de Estimativas de Emissões de Gases de Efeito Estufa - 2018. < http://plataforma.seeg.eco. br/total_emission\# > . Acesso em: 10/10/2020.

SEPLAG (Secretaria de Estado de Planejamento e Gestão. Governo de Mato Grosso), 2014. http://www. seplag.mt.gov.br/images/files/responsive/Planejamento/ INFORMACOES MUNICIPAIS/BREVE_HISTORICO DOS_MUNICIPIŌS_MATOGROSSENSESS.pdf. Acesso em: $\overline{15} / 06 / 2020$.

Silvestrini RA, Soares-Filho BS, Nepstad D, Coe M, Rodrigues $\mathrm{H}$ \& Assunção R. Simulating fire regimes in the Amazon in response to climate change and deforestation. Ecological Applications, 21(5), 2011, pp. 1573-1590. 2011 by the Ecological Society of America. https://doi.org/10.1890/10-0827.1. Acesso em: 24/05/2020.

WMO (World Meteorological Organization). Calculation of monthly and annual 30-year standard normals: WMO-TD/No. 341. World Meteorological Organization, Geneva, 1989.

Biodiversidade Brasileira - BioBrasil.

Fluxo Contínuo

n. 3, 2021

http://www.icmbio.gov.br/revistaeletronica/index.php/BioBR

Biodiversidade Brasileira é uma publicação eletrônica científica do Instituto Chico Mendes de

Conservação da Biodiversidade (ICMBio) que tem como objetivo fomentar a discussão e a disseminação de experiências em conservação e manejo, com foco em unidades de conservação $e$ espécies ameaçadas.

ISSN: 2236-2886 